\title{
Cryptococcal meningitis in HIV-negative patient with liver cirrhosis due to hepatitis C
}

Dear Editor,

Before AIDS, cryptococcal meningitis, a disease caused by the basidiomycete fungus Cryptococcus neoformans, occurred at an incidence rate of one case per one million person-years. ${ }^{1}$ After AIDS, transplants and the use of immunosuppressive therapies, the incidence rate increased tenfold. ${ }^{1,2}$

We report a case of a 46-year-old male with a history of headache, confusion, nausea and vomiting for one month. The patient also had type 2 diabetes mellitus, in use of metformin, with good control, and liver cirrhosis due to hepatitis C. Treatment with pegylated interferon and ribavirin had begun two months before clinical presentation. After the second dose of interferon, pancytopenia was observed (hemoglobin: $10.9 \mathrm{~g} / \mathrm{dL}$, leukocytes: 3,660 cells/ $\mu \mathrm{L}$, platelets: $36,000 / \mu \mathrm{L})$, decompensated cirrhosis and diabetes mellitus, characterized by moderate ascites and grade 3 hepatic encephalopathy, which overlapped the clinical symptoms described above, despite treatment with therapeutic doses of furosemide, spironolactone, lactulose, NPH and regular human insulin. Patient was listed for liver transplantation. Model of End-Stage Liver Disease (MELD) score equals 14 points.

At presentation, cerebrospinal fluid (CSF) showed 21 cells $/ \mathrm{mL}$, total protein $=96 \mathrm{mg} / \mathrm{dL}$, glucose $=34 \mathrm{mg} / \mathrm{dL}$ (serum glucose: $290 \mathrm{mg} / \mathrm{dL}$ ), positive India ink staining and cryptococcal antingen titer greater than $1 / 1,024$. Culture for C. neoformans was positive in the CSF, but negative in blood, bone marrow and ascites. Central nervous system computed tomography and magnetic resonance imaging has showed only mild supratentorial hydrocephalous. Patient evolved with intracranial hypertension (the first opening pressure: $36 \mathrm{cmH}_{2} \mathrm{O}$ ), without improvement by lumbar puncture. Ventriculoperitoneal shunt was performed after two units of apheresis platelets transfusion.
The patient was treated with amphotericin B lipid complex $5 \mathrm{mg} / \mathrm{kg} /$ day for 30 days and 5 -fluorocytosine $100 \mathrm{mg} / \mathrm{kg} /$ day for 14 days until three negative cultures for $C$. neoformans were obtained. During treatment, he presented elevated creatinine $(2.0 \mathrm{mg} / \mathrm{dL})$, but without requiring dialysis. There was no worsening of pancytopenia during treatment. Strict monitoring of renal and liver function and bone marrow was performed.

Before starting fluconazole $800 \mathrm{mg} /$ day, the patient has presented bloodstream infection associated with central venous catheter by Enterococcus faecalis, and despite appropriate treatment with vancomycin for 10 days, the patient progressed with septic shock syndrome and multiple organ dysfunction syndrome (MODS) and died 40 days after admission.

Cryptococcosis affects the central nervous system in $51.3 \%$ of HIV-negative patients, after the introduction of azole agents in the market, and in 39\% of patients with cirrhosis, as suggested by the two largest studies in the literature. ${ }^{3,4}$ The peritoneum is the site most associated with cryptococcal infection in cirrhotic patients (45\%). ${ }^{4}$

In $306 \mathrm{HIV}$-negative patients, the underlying causes were steroids $(28 \%)$, organ transplant (18\%), chronic shortage of organs (liver, respiratory and renal) (18\%), neoplasm (18\%), rheumatic diseases (13\%), and unknown risk factor (22\%). ${ }^{3}$ Malnutrition, in addition to compromised phagocytosis, immunoglobulins and cellular immunity result in increased risk of cryptococcosis in cirrhotic patients. ${ }^{4}$ The case fatality rate associated with cryptococcosis in cirrhotic patients is $83 \%, 92 \%$ of which attributed to cryptococcosis. In 53\% of cases, death occurs in the second week of diagnosis. Culture positivity is $67 \%$ and India ink in around $50 \%$ in cirrhotic patients and HIV-negative, respectively. ${ }^{1,2}$
Authors

Érique José Peixoto de

Miranda $^{1}$

Luiz Guilherme

Gonçalves ${ }^{1}$

Francisco Oscar de

Siqueira França ${ }^{2}$

${ }^{1} \mathrm{MD}$, Resident Physician, School of Medicine,

Universidade de São Paulo (USP), SP, Brazil

${ }^{2}$ Professor, Department of Infectious Diseases, School of Medicine, USP, SP, Brazil

Submitted on: 09/27/2010 Approved on: 09/29/2010

Correspondence to: Érique José Peixoto de Miranda

Rua Enéas de Carvalho Aguiar, 255,

Cerqueira Cesar 05403-000

São Paulo, SP, Brazil erique@fcm.unicamp.br

We declare no conflict of interest.

(C)2011 Elsevier Editora Ltda. All rights reserved. 
This case was a rare example of cryptococcosis without dissemination in an HIV-negative patient. Combined treatment with fluorocytosine and amphotericin B lipid complex resulted in CSF sterilization, but a bacterial bloodstream infection, in spite of appropriate treatment, was the cause of death, which might be attributed to immunosuppression caused by underlying disease.

\section{REFERENCES}

1. Jackson A, Powderly WG. Criptococcal infection. In: Portegies R, Bergers JR. Handbook of Clinical Neurology - vol. 85, 3th edition. Elsevier, 2007.

2. Bicanic T, Harrison TS. Cryptococcal meningitis. Brit Med Bull 2004; 72(1):99-118.

3. Pappas PG, Perfect JR, Cloud GA et al. Cryptococcosis in human immunodeficiency virus-negative patients in the era of effective azole therapy. Clin Infect Dis 2001; 33(5):690-9.

4. Singh N, Husain S, De Vera M et al. Cryptococcus neoformans Infection in patients with cirrhosis, including liver transplant candidates. Medicine (Baltimore). 2004; 83(3):188-92. 